\title{
Articulación de semántica y pragmática. Los dos principios subyacentes a todas las operaciones lingüísticas
}

\section{Articulation of Semantics and Pragmatics. The Two Principles Underlying All Linguistic Operations}

\author{
José Luis Iturrioz Leza \\ Departamento de Estudios en Lenguas Indígenas \\ Universidad de Guadalajara, México \\ jliturrioz@yahoo.com.mx \\ Ana Line Martínez Sixto \\ Departamento de Estudios en Lenguas Indígenas \\ Universidad de Guadalajara, México \\ analinems@yahoo.com.mx
}

Resumen

La semántica y la pragmática son dos componentes operativos igualmente necesarios que actúan de manera circular y complementaria en todos los niveles de organización de la lengua. Las lenguas funcionan como sistemas dinámicos porque no están constituidas solamente por reglas semánticas formales y abstractas, sino también por reglas pragmáticas que coarticulan en la conducta verbal el significado con el conocimiento y las situaciones comunicativas. Las lenguas recurren a técnicas diferentes para la misma función, por ejemplo para la aprehensión de los sonidos, olores, colores y sabores. En la técnica de etiquetado específico, predominante en algunas lenguas, prevalece el componente semántico, organizando en el léxico paradigmas extensos de términos específicos que se cargan en la memoria, mientras que otras lenguas como mi?phaa dan preferencia a otras técnicas como el etiquetado genérico basada en reglas inferenciales que actúan de acuerdo con el contexto. Aquéllas son semantizantes o paradigmatizantes, éstas pragmatizantes o contextualizantes. De ahí se derivan consecuencias fuertes para la lexicología, la tipología, así como para la traductología.

Palabras clave: pragmática, semántica, significado inherente, técnicas inferenciales

Abstract

Semantics and pragmatics are two equally necessary operational components that act in a circular and complementary way at all levels of language organization. 
Languages function as dynamic systems because they are constituted not only by formal and abstract semantic rules, but also by pragmatic rules that co-articulate in verbal activity inherent meaning with practical knowledge and communicative situations. Languages use different techniques for the same function, for example for the apprehension of colors, sounds, smells and tastes. In some techniques such as specific labeling, predominant in wixárika, Spanish and German, the semantic component prevails, organizing in the lexicon extensive paradigms of specific terms that are loaded in memory, while other languages such as mi? phaa give preference to other techniques such as generic labeling based on inferential rules that act according to context. This implies strong consequences for lexicology, typology as well as for translation.

KEYwORDS: inherent meaning, inferential tecniques, wixárika, miphaa, basic terms, linguistic operations

FECHA DE RECEPCIÓN: 30/06/2020

FECHA DE ACEPTACIÓN: 07/10/2020

\section{Nota histórica}

La semántica y la pragmática han seguido rutas de desarrollo divergentes y se han centrado en diferentes dominios de organización de la lengua. Durante mucho tiempo se desconocieron y se disputaron el protagonismo en la descripción de la misma. El campo preferencial de la semántica ha sido el léxico, el de la pragmática el discurso.

En las teorías de orientación formal, como el estructuralismo y la gramática generativa, la pragmática no desempeñó ningún papel relevante; se concedió la primacía al sistema abstracto. Por el contrario, en las teorías orientadas a la lengua como actividad, la pragmática fue considerada como el componente central, origen de todas las categorías y razón de ser de los fenómenos semánticos y gramaticales. La pragmática se desarrolló aparte en disciplinas como la filosofía, la sociolingüística y la psicología, ganando terreno lentamente en los estudios sobre el texto o la adquisición de la lengua. El interés de Bruner por la adquisición de la lengua no estaba en los aspectos formales de la gramática, sino en la pragmática: cómo aprenden los niños a usar el lenguaje y, más específicamente, cómo aprenden a interactuar para "lograr una atención recíproca y el manejo de 
acciones en común con otra persona por medio del uso del lenguaje" (1983, p. 11). Al "explorar las funciones que el lenguaje cubre en diferentes contextos y la forma en que estas funciones se desarrollan", Bruner (1983, p. 11) tiene en cuenta la sensibilidad al contexto y las formas de interacción entre la madre (o los adultos en general) y el niño, la contextualización del pensamiento y del habla, así como la incidencia de reglas culturales. Es el camino seguido por Tomasello en sus trabajos sobre la adquisición. (Ver en especial, Tomasello, 2002). En Iturrioz (1998) se destaca la importancia de un discurso pragmático en las fases iniciales de comunicación verbal que emana de la regulación de las acciones prácticas.

En la lingüística del texto, la coherencia se ubicó primero en el terreno de la semántica, pero poco a poco fue cediendo espacio a la pragmática, delimitando cada vez con más precisión ambos dominios (Viehweger, 1989; Harweg, 1989; y Lundquist, 1991).

En la lingüística funcional se ha venido integrando la pragmática en la descripción sintáctica como un complemento de la semántica. En sintaxis ha sido muy fructífera la distinción de roles semánticos y roles pragmáticos. Comrie (1981) dedica una sección del capítulo 3 a los primeros, y la siguiente a los segundos. Los primeros se ubican en la semántica proposicional, mientras que los segundos pertenecen al nivel de organización llamado sintaxis. En la lingüística natural desarrollada en Viena bajo la dirección de Dressler se creó una morfopragmática junto a la morfosemántica (Dressler, 1990; Dressler y Barbaresi, 1987, 1993), y en el nivel del texto se acabó dando tanta importancia a la coherencia pragmática como a la coherencia semántica. En Iturrioz (2013) la coherencia pragmática es vista en una relación de articulación con la coherencia semántica y la coherencia cognitiva.

Pero es hasta la lingüística operacional, desarrollada en un proyecto de investigación de largo plazo en la Universidad de Colonia bajo el nombre UNITYP (Universales y Tipología) que se persigue la integración de semántica y pragmática en una visión circular, de complentariedad y mutuo condicionamiento, considerando que son dos componentes que se coarticulan en todos los niveles de organización de las lenguas, desde la fonología hasta el texto o discurso, en proporciones inversamente pro- 
porcionales. Iturrioz (1986) describe el desarrollo de este modelo desde principios de los años setenta hasta ese momento dando cuenta del papel fundamental que desempeñan los dos principios de semanticidad y pragmaticidad en todas las operaciones lingüísticas y en cada una de las técnicas de que se componen. Los principios operacionales de semanticidad y pragmaticidad son la manifestación en el lenguaje de las invariantes funcionales que de acuerdo con la epistemología genética organizan todo tipo de conducta adaptativa: la asimilación es responsable de la interiorización de la acciones y su organización en un sistema abstracto, mientras que la acomodación da cuenta de que este sistema abstracto no se desconecta de la conducta práctica (referencialidad, aplicación al contexto, situacionamiento) y del conocimiento del mundo (cognición) (Piaget 1974, 1984).

Este artículo es un breve anticipo de un libro de nuestra autoría titulado Las lenguas y los sentidos, donde acometemos la tarea de mostrar cómo se complementan ambos componentes en la construcción de cuatro dominios sensoriales (sonido, color, olor y sabor) en cuatro lenguas (castellano, alemán, wixárika y mi'phaa). En cada lengua estudiamos los cuatro dominios sensoriales desde dos perspectivas complementarias:

1. intralingüística, comparando dentro de cada lengua los cuatro dominios sensoriales, $y$

2. interlingüística, comparando las cuatro lenguas para cada dominio sensorial.

\section{Obtención de datos}

Nuestra investigación no se restringe a un solo campo semántico ni a la capa más básica del léxico, sino que tiene en cuenta todos los recursos de que dispone cada lengua en cada dominio. Si preguntamos a un hablante normal de qué recursos dispone su lengua para hablar de colores, olores, sabores o sonidos, buscaría en su memoria o en un diccionario las palabras que se refieren a estos cuatro tipos de sensaciones. Durante mucho tiempo los lingüistas no han ido más allá, y los especialistas de otras disciplinas afines tampoco. En su estudio antropológico, Berlin y Kay (1969) ni siquiera tienen en 
cuenta todo el vocabulario de que disponen las lenguas comparadas, sino que se limitan a lo que llamaron términos básicos de color.

Para llevar a cabo esta investigación se requería reunir desde una definición lingüísticamente adecuada todos los términos básicos (técnica de etiquetado específico), pero también indagar qué uso hacen las cuatro lenguas de otras técnicas nominativas o de formación de términos como la transferencia, la metáfora, la metonimia, derivación, composición, incorporación etc. (fase gramatical) hasta completar una escala de opciones llamadas técnicas, entre ellas técnicas no nominativas (analíticas) hasta componer una operación lingüística completa. En la técnica de etiquetado específico había que llegar a un inventario exhaustivo, en las demás había que averiguar rasgos operativos como la productividad. La metodología para la obtención de datos no podía ser la misma para las cuatro lenguas, dos de las cuales están bien documentadas y las otras dos no. Para castellano y alemán se revisaron todos los grandes diccionarios (DLE, DEM, DEA; Wahrig, Duden, Langenscheidt), la base de datos CREA y otras fuentes documentales, técnicas y literarias. El 75\% de los términos reunidos están en $\mathrm{La}$ historia interminable de Michael Ende.

Para $\mathrm{mi}^{22}$ phaa $^{2}$ utilizamos tres fuentes de datos. La primera fueron los textos disponibles. Los resultados fueron muy exiguos. En los libros para la primaria de la SEP no se encontró ni un solo término relacionado con los sonidos. La revisión de las 800 páginas de la traducción de la biblia al $\mathrm{mi}^{22}$ phaa ${ }^{2}$ realizada por el ILV (Instituto Lingüístico de Verano) dio como resultado 8 términos básicos. La segunda fuente de datos fueron largas conversaciones grabadas donde los participantes, muchos monolingües, debían conversar sobre los sonidos de fenómenos naturales, de animales o de instrumentos. También aquí la cantidad de términos básicos hallados fue muy exigua. La tercera fuente consistió en obtener correspondencias para los términos básicos inventariados del español; la tarea consistía en expresar en miphaa el significado de todos los términos reunidos en español, y la mayoría de las traducciones resultaron ser expresiones complejas, compuestas de más de una palabra. Para wixárika disponemos de una gran cantidad de textos con los que hemos documentado la lengua a lo largo de 30 años, y del léxico huichol, compuesto unos 130,000 
artículos (casi el doble que el DLE). Además se llevó a cabo una investigación lexicográfica exhaustiva en la comunidad en largas y reiteradas estancias de campo, logrando un número de términos básicos bastante superior al de alemán y español.

El objetivo general era llevar a cabo una comparación de las cuatro lenguas desde una perspectiva más amplia que los términos básicos ampliando la investigación a una gama de técnicas alternativas, pasando del concepto de campo semántico al más abarcador de dominio operacional, de los términos básicos a las operaciones lingüísticas.

\section{Operaciones lingüísticas}

Una operación lingüística es un conjunto de técnicas complementarias destinadas a una misma función. Se han propuesto y descrito operaciones para la función de INDividuación, DETerminación (o identificación), POSesión, LOCalización, NOMinalización, PARTicipación, ATRibución, y otras. Para cada dominio funcional, las lenguas hacen una selección particular de técnicas y les asignan una relativa prominencia o tipicidad; en el nivel de las técnicas opera la tipología, y en el nivel de las operaciones se establecen las funciones y los principios universales. El esquema siguiente representa una operación lingüística.

Las técnicas se ordenan en una escala de creciente complejidad morfosintáctica, o sea de complejidad morfológica de los términos, desde las etiquetas básicas hasta la incorporación sintáctica, y de complejidad sintáctica en el resto de la escala. Se parece a la escala de descriptividad, definida originalmente por Seiler (1976), pero nuestra interpretación se distingue en dos aspectos fundamentales. En primer lugar, abarca más que técnicas de nominación; a partir de la incorporación sintáctica, ya no son términos inventariables, sino construcciones sintácticas que pueden identificar los sonidos sin nombrarlos. En segundo lugar, aunque la cantidad de información crece hacia la derecha por la suma de los significados de todos los componentes, la información específica relativa al dominio en cuestión decrece gradualmente a la par que se fortalece el componente pragmático. 
José Luis Iturrioz Leza y Ana Line Martínez Sixto

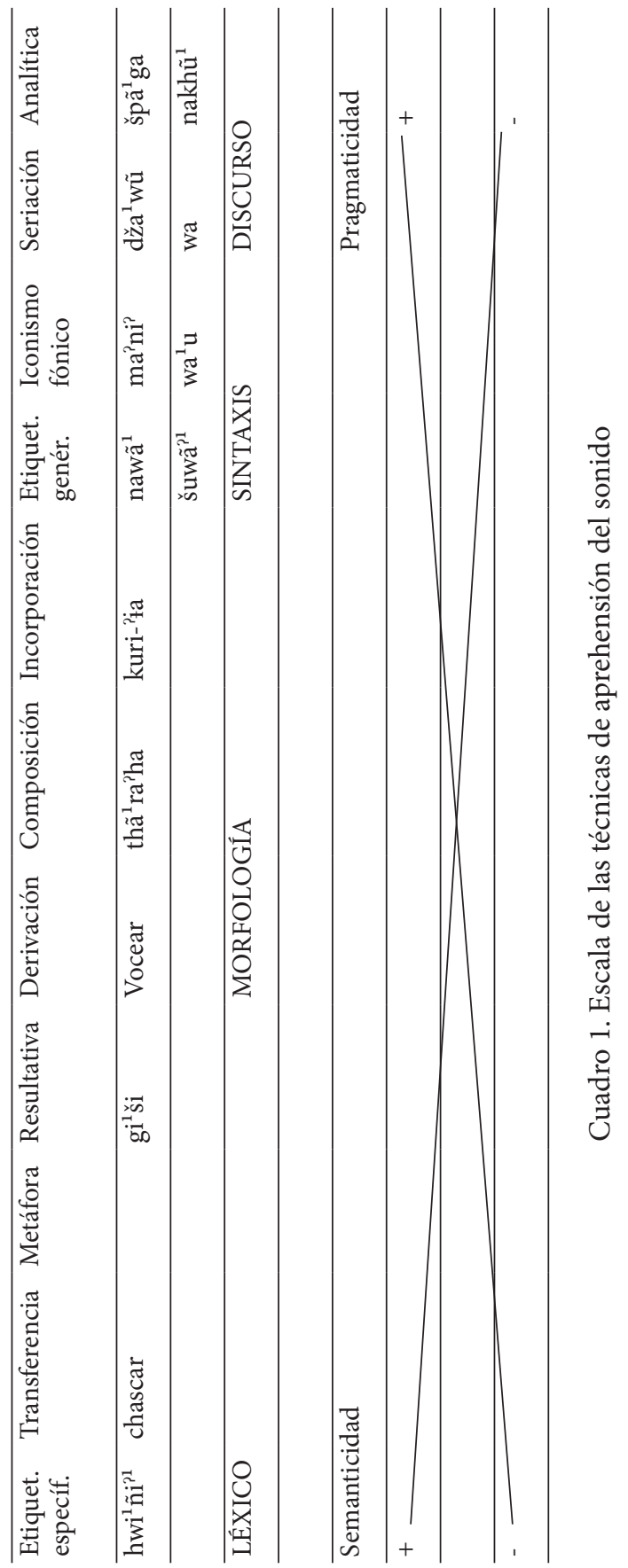


Las expresiones lingüísticas cumplen su función comunicativa obedeciendo a estos dos vectores esenciales de la dinámica de todo sistema comunicativo, que operan en forma complementaria, interdependiente, bidireccional o circular; la pragmática acomoda el significado a las situaciones, la semántica fija en esquemas abstractos esa puesta en correlación de las expresiones con las situaciones. En la comunicación tiene lugar una continua adaptación mutua. La acomodación pragmática parte del significado de las expresiones y puede, de rebote, modificar o diversificar el significado de éstas. Muchos cambios semánticos están basados en asociaciones inferenciales. Las reglas de la pragmática son también parte de la lengua entendida como actividad. En el enunciado la nave descendió suavemente sobre Marte, componemos para nave un significado en el que están ausentes rasgos que no corresponden al discurso de la navegación espacial. Pero la pragmática no sólo contribuye a la eliminación de algunos rasgos, sino que también puede añadir otros, como ocurre en mi'phaa en varias de las técnicas.

En el cuadro siguiente ilustramos con un ejemplo cada una de las técnicas:

\begin{tabular}{|c|c|c|}
\hline 1 & 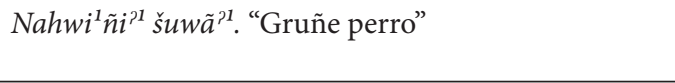 & $\begin{array}{l}\text { Etiquetado } \\
\text { específico }\end{array}$ \\
\hline 2 & $\begin{array}{l}\text { Oler mal ciertas partes del cuerpo: } \\
\text { Le cantan los sobacos }\end{array}$ & Transferencia \\
\hline 3 & Barruntar. Al obispo el texto le huele a herejía & Metaforización \\
\hline 4 & Ndžahwa1-ska "voz ensordecedora" & Derivación \\
\hline 5 & Fern-sprechen "lejos hablar" (hablar por teléfono) & Composición \\
\hline 6 & Wix. kuri-'ia "oler a podrido" (literal podridoler) & Incorporación \\
\hline 7 & Nawã ${ }^{1}$ šuwã $\tilde{a}^{11}$ " Suena perro" (= ladrar) & $\begin{array}{l}\text { Etiquetado } \\
\text { genérico }\end{array}$ \\
\hline 8 & $\begin{array}{l}\text { Niši } i^{1} g u \text { škwi } i^{1} y a . \\
\text { "Las ramitas se quebraron / crujieron" }\end{array}$ & $\begin{array}{l}\text { Resultativa } \\
\text { (metonimia) }\end{array}$ \\
\hline 9 & El perro hizo guau guau & $\mathrm{V}_{\text {gen }}+$ icono fónico \\
\hline 10 & Iwã ${ }^{1} e^{\text {'ndita}}$ 'ha. "Suena barrenar" & Seriación \\
\hline 11 & $\begin{array}{l}\text { Nawã } \tilde{a}^{1} \text { špa } \tilde{a}^{1} g a \text { hmaa nakhu } u^{1} \tilde{n} a w \tilde{u}^{?} \check{s}^{3} b u^{3} n a^{p 1} i i^{1} y a^{p^{3}} \\
\text { suena agitar con pies manos persona en agua } \\
\text { (= chapotear) }\end{array}$ & Técnica analítica \\
\hline
\end{tabular}

Cuadro 2. Técnicas de aprehensión del sonido 
En la mitad izquierda (o superior) de la escala están las técnicas pertenecientes al léxico, donde tiene más peso la semántica, mientras que a partir de la incorporación la pragmática asume un papel preponderante. A partir del etiquetado genérico, los términos no aportan casi información específica sobre el sonido, lo que se suple con información sobre la fuente, sobre el proceso que genera el sonido, sobre la emisión / recepción del sonido, o sobre las circunstancias en que se produce. El punto de inflexión de esta escala se ubica en la técnica de incorporación, que se encuentra a caballo entre la formación de términos y la pragmática discursiva (Mithun, 1984, 1986; Iturrioz, 2001).

También tomamos en cuenta la complejidad vertical o paradigmática, cuando en la escala original de Seiler (1976) sólo se consideraba la complejidad sintagmática.

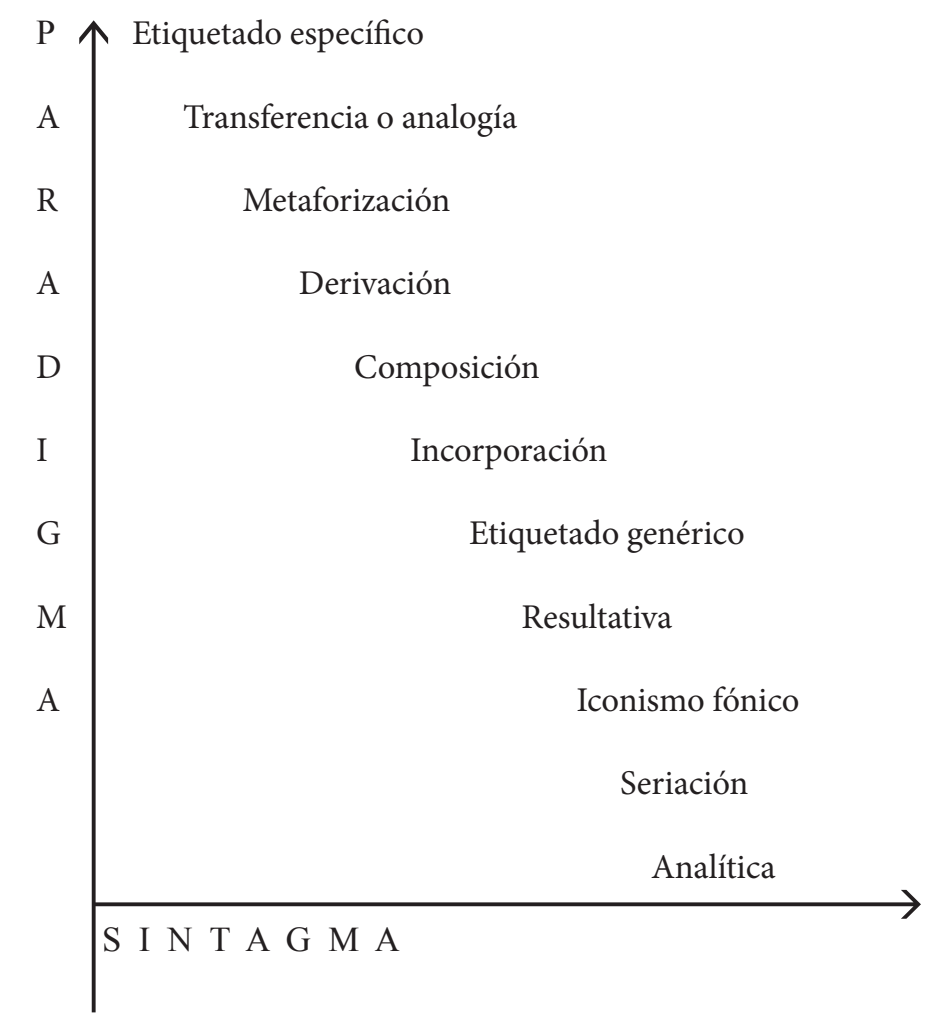

Cuadro 3. Escala de las técnicas en un espacio bidimensional 
Una construcción será tanto más compleja semánticamente cuanto mayor sea el número y la especificidad de sus elementos en el eje paradigmático (vertical en el nuevo esquema). La complejidad semántica inherente de los términos básicos aumenta en la medida en que crece el inventario del campo semántico. La complejidad global de una expresión está determinada por ambos ejes. Ambos tipos de complejidad tienden a crecer de manera inversamente proporcional: si el término básico es semánticamente rico (específico), se necesitan menos recursos sintácticos para identificar el sonido; los términos básicos muy específicos son más compactos y casi no se pueden combinar con otras palabras relacionadas con el sonido sin riesgo de redundancia, pero cuando los términos básicos sean muy genéricos mayor será la necesidad de aportar información acerca de la fuente o las circunstancias de la situación en que se produce el sonido, y viceversa.

Cuanto menor es la cantidad de información específica sobre el sonido, mayor debe ser el grado de actividad inferencial (pragmática) que se requiere para identificar el sonido. Cuanto más genérico sea un término básico, más recursos pragmáticos se necesitarán para identificar un sonido, y viceversa. Si decimos se oye un animal, la falta de información inherente se debe suplir con información obtenida por la observación y el conocimiento del medio, es decir a través de inducciones. Si decimos están croando las ranas, hay mucha redundancia, ya que croar identifica por sí mismo a la especie. Si decimos toda la noche se oyeron los perros, lo más probable es que se trate de ladridos debido a nuestro conocimiento del mundo, pero podrían ser otros sonidos. En mi'phaa, por el contrario, nawa ${ }^{1}$ šuwã $^{\text {?1 }}$ (literalmente suena perro) sólo se puede entender como el perro está ladrando. ¿De dónde procede la información adicional relativa al sonido no codificada en las dos palabras? La respuesta de una inducción a partir del conocimiento del mundo que comparten el hablante y el oyente no es suficiente. Ese conocimiento del mundo lo comparten también los hablantes de castellano, alemán o wixárika, pero éstos no tienen que entender necesariamente se oyeron los perros como ladraron los perros. Está implicada también la pragmática mediante una regla que viene a decir: un verbo genérico de sonido se combina con la designación de la especie 
para referirse a la voz característica de la misma: $w \tilde{a}^{1}$ se entiende como ladrar cuando se predica de perros y como croar cuando se predica de ranas. Por esta regla, en mi'phaa se construye contextualmente lo que en otras lenguas se construye en el léxico.

En la técnica de etiquetado específico el sujeto puede ser genérico, mientras que en la técnica de etiquetado genérico el sujeto tiende a ser específico. Debe haber un equilibrio entre la especificidad de la información aportada por los términos de sonido y la información complementaria añadida por el resto de la cláusula. En esta contraposición se sustenta la escala de las técnicas: la carga semántica de los términos frente a las reglas pragmáticas que permiten inducir información a partir del entorno lingüístico y situacional. No son los sonidos los que se describen con creciente explicitud porque en la segunda mitad de la escala la descripción no es tanto del sonido como de la fuente o de la situación en que se produce.

\section{Semántica y pragmática contrastivas}

No es fácil acercarse a la organización del significado en una lengua como mi'phaa sin proyectar sobre ella la estructura de lenguas más familiares al observador. Para que la posición del observador (hablante de castellano, alemán, huichol o náhuatl) no transforme el objeto de análisis (mi?phaa), debemos llevar a cabo un ejercicio riguroso de semántica y pragmática contrastivas. Un acercamiento que parta de preguntas como

1. ¿Cómo se dice en miphaa "ladrar"?
2. ¿Cómo se traduce al castellano ši ${ }^{1}$ gu?

puede generar respuestas poco justificables desde un punto de vista teórico y práctico; por ejemplo, para quien desee llevar a cabo una traducción. Las respuestas podrían ser:

1. En mi'phaa no existe un término específico para "ladrar", pero el verbo wã ${ }^{1}$ se puede traducir al castellano como ladrar, mugir, rebuznar, relinchar, cacarear, berrear, etcétera. 
2. El significado de $\mathrm{si}^{1}$ gu se puede parafrasear como perder con facilidad su unidad un objeto. En castellano no hay un término que traduzca exactamente esta idea; el más aproximado es frágil, pero según los casos se podría elegir quebrarse, cascarse, machacar, triturar, chascar, ronzar, resquebrajarse, agrietarse, cuartearse, rajarse, henderse, craquelar, saltar, desgranarse, desmoronarse, sufrir un deslave o derrubio, añadiendo en todos los casos con facilidad, que no es un rasgo inherente de ninguno de ellos.

Un cambio de pregunta podría producir una inversión de la sorpresa. Para qué necesita el castellano tantos términos si lo mismo se puede decir de manera más económica, con menos redundancia, sirviéndose de un verbo que expresa la idea general y construyendo los significados específicos a partir del contexto y la capacidad inferencial. Si juzgamos al castellano desde el mi'phaa, tenemos que aceptar que es una lengua extraña. Se requiere abandonar los planteamientos y métodos que generan ese tipo de preguntas y de respuestas, y adoptar un marco teórico más explicativo, así como herramientas descriptivas que permitan apreciar la complementariedad y la equivalencia funcional en la diversidad estructural. El método contrastivo debe ayudar a entender y explicar las profundas diferencias que separan a las lenguas, pero también la equivalencia en la diversidad. Una concepción adecuada del lenguaje es la que permite explicar la variación de las lenguas, sin hacer aparecer ni a unas ni a otras como extrañas, raras o exóticas.

En mi’phaa prevalece el principio de no construir un campo semántico también en el dominio de los sonidos, mientras que las otras tres tienden en diferentes grados a lo contrario. Con otras palabras, en mi?phaa predomina la técnica de etiquetado genérico y en las otras la técnica de etiquetado específico. A partir del etiquetado genérico se identifica un sonido no a partir del significado léxico, sino a través de inferencias pragmáticas; no hay un término que aporte información específica sobre un sonido, éste se debe inferir de la fuente mencionada. En la seriación, la información sobre el sonido es genérica y redundante, y la téc- 
nica analítica es más una descripción de la situación en que se genera el sonido que una descripción del sonido mismo.

Del etiquetado específico se nutre sobre todo el huichol, en alemán son además muy típicas la composición y la derivación, y en castellano es prominente la derivación (olisquear, olfatear, oloroso, sabroso, azulado, amarillento, negruzco). En mi? phaa la técnica menos prominente en los cuatro dominios es el etiquetado específico y las más prominente el etiquetado genérico, la técnica resultativa, la seriación y la técnica analítica, todas ellas ubicadas en la parte derecha de la escala.

\section{De los términos básicos a la técnica de etiquetado específico}

Para reunir los términos básicos de una lengua se requiere una definición lingüística clara y fácilmente aplicable de básico como la siguiente:

1. Un término básico debe ser simple en la expresión, sin complejidad morfosintáctica, no derivado ni compuesto ni apositivo, etcétera.

2. Pertenece primariamente al dominio semántico en cuestión; no debe mediar una transferencia de otro dominio sensorial (limón agrio $\rightarrow$ sonido agrio). Púrpura, rosa, anaranjado, orange, purple no son básicos; antes que un color, designan sustancias o frutas.

3. No debe ser resultado de una metáfora (carácter agrio), analogía o metonimia.

4. No deben ser resultado de una inferencia (en mi?phaa $\check{s}^{1} g u$, con el sentido de crujir).

5. Su significado no debe ser un caso especial de un significado más general ( $e^{21} n i^{1}$, "hacer; sonar").

6. No debe designar una variante o matiz de otro término, que necesariamente figura en su definición.

Rojo, blanco, azul y amarillo son términos básicos porque cumplen todas las condiciones, pero no rojizo, blancuzco, blan- 
quecino, azulado ni amarillento, porque son derivados; tampoco concha, plata, púrpura, rosa, burdeos ni turquesa porque no pertenecen primariamente al dominio de los colores. Según esta definición, la palabra inglesa orange no pertenece primariamente al campo de los colores, sino que resulta de una transferencia: el significado primario tiene que ver con el árbol y el fruto. La palabra Burdeos designa primariamente una ciudad francesa; en segundo lugar, el vino de la región (nos tomamos un burdeos) y, en tercer lugar, el color típico del vino de Burdeos (compré una camisa burdeos). Turquesa es primariamente el nombre de un mineral.

El esquema que sigue concentra los datos sobre los términos básicos de los cuatro dominios en cada una de las lenguas investigadas. El dominio donde más divergen los datos es el de los sonidos, en tal medida los resultados obtenidos contradicen frontalmente las conclusiones a que llegaron estos autores, pero al menos podemos alegar que éstas no resultan aplicables a los términos de sonidos.

\begin{tabular}{lllll}
\hline Lengua & Colores & Olores & Sabores & Sonidos \\
\hline Wixárika & 6 & 2 & 4 & 800 \\
\hline Alemán & 7 & 10 & 5 & 250 \\
\hline Castellano & 8 & 13 & 6 & 117 \\
\hline Miphaa & 8 & 6 & 4 & 13 \\
\hline
\end{tabular}

Cuadro 4. Términos básicos de color, olor, sabor y sonido en las cuatro lenguas

En el dominio de los colores, sabores y olores, las cuatro lenguas son pobres en vocabulario básico. En los dominios de los olores y los sabores, las cuatro lenguas utilizan la misma técnica, que llamamos etiquetado genérico: huele a nardos, sabe a verdolagas; al. es stinkt nach Mist, es schmeckt nach Olivenöl; wi. pitiuketsì'ta, "huele a pescado"; payïna'ta "sabe a tuna"; $m i$. ndathayũ kafe "huele a café". De manera parecida se comporta el mi?phaa en el dominio de los sonidos: nawa ${ }^{1} \check{s} u w \tilde{a}^{\text {?1 }}$, "suena perro" (ladrar), donde las otras lenguas recurren a la técnica de 
etiquetado específico. En este dominio hay una gran discrepancia numérica: las cuatro lenguas utilizan un paradigma de términos básicos cuya amplitud es muy variable, desde 13 términos en mi?phaa, hasta aproximadamente 800 en wixárika.

Si interpretamos estos datos como se ha venido haciendo con los términos básicos de color a partir de Berlin y Kay (1969), mi?phaa se hallaría en una etapa primitiva de desarrollo (en los cuatro dominios), mientras que wixárika sería una lengua mucho más desarrollada social y tecnológicamente que la alemana, la inglesa o la "castellana" en el dominio de los sonidos. La tesis de Berlin y Kay sobre la determinación extralingüística sólo tiene en cuenta los términos básicos de un único dominio, pero todas las lenguas poseen en todos los dominios recursos alternativos, complementarios y con diferente prominencia.

Palabras como crascitar, crotorar, ronronear, ladrar, barritar o gruñir designan voces específicas de especies animales. En wixárika muchos de los términos básicos pueden ser sumamente específicos: kaxanarika, "ruido que se produce al pisar la hojarasca o ramaje seco"; kuxanarika, "sonido que se produce al arrastrar un cuerpo"; karaminarika, "sonido de piernas mojadas que se produce cuando se les echa jabón al bañarse"; kararikarika, "sonido seco como el que se produce con las cáscaras de guajes, pezuñas de venado o bolitas de dulce de amaranto al chocar entre sí"; karaunarika, "sonido de jícaras recién cortadas en el montón y al tocarlas"; kwararikarika, "sonido de pantalones mojados". En castellano, el perro ladra, el burro rebuzna, el caballo relincha, el rinoceronte barrita, la gallina cacarea, la cigüeña crotora; en alemán, el asno bräht, el caballo wiehert, el gallo kräht, la gallina gackert, el pato schnattert, el perro bellt, el cerdo grunzt, un enfermo stöhnt, etcétera.

\section{Otras técnicas léxicas}

Hay que ir más allá de los términos básicos, explorando de qué otros recursos disponen las lenguas para aprehender los sonidos, colores, sabores y olores. No sólo de términos básicos viven las lenguas. 
Las extensiones (gruñir, dicho de seres humanos, puertas o tripas; kwiká dicho de muchas especies de aves) permiten abarcar otros referentes dentro del mismo dominio sensorial; ulular se dice de los lobos y perros, pero también se dice de un búho, del viento o del mar en virtud de cierto parecido acústico entre los sonidos. Los aullidos o alaridos de perros y lobos son el prototipo, y el parecido con el mismo determina la ampliación; de ahí la definición "producir un sonido parecido al del viento" (DLE).

Las transferencias conllevan un cambio de un dominio a otro (chascar pasa de "triturar, ronzar algún alimento quebradizo" a "hacer ruido al triturar") sobre la base de una analogía o iconicidad diagramática entre ambos o a una relación metonímica.

La metaforización se basa en una analogía o transferencia vertical entre niveles cognitivos. Cuando en español decimos que un asunto "levanta mucho polvo", se trata de una metáfora; se produce un cambio del plano sensorial de los eventos físicos al plano más abstracto de los eventos conductuales, que de hecho se llevan también a cabo sin sonidos, en la prensa escrita o en internet. Por el contrario, en $n a^{2} n i y o o r s e ~ d i$, "el toro hace polvo" (escarbando con las patas delanteras, mi?phaa) no se produce una metáfora, su relación con bramar y estar en celo no es parte del significado de $e^{2}$ niyoo ni de la palabra $\check{s}^{1}{ }^{1} i$, se trata de una asociación que no se da con independencia de la acción de levantar polvo realizada por el toro; el verbo no deja de significar "levantar polvo", no se trata de un significado léxico nuevo.

De ' $k h u^{3}$ "morder" se deriva ' $k h u^{3} m i^{1} n a^{\text {', }}$ que según la fuente designa sonidos que en español son descritos con términos diferentes: ' $k h u^{3} m i^{1} n a^{?} i^{1} \tilde{n} u^{\text {? }}$ (diente), "carrasquear" (crujir o rechinar entre los dientes una sustancia algo dura, seca y quebradiza); "castañetear" (sonar los dientes, golpeando, dando los de una mandíbula contra los de la otra); "chirriar, rechinar"

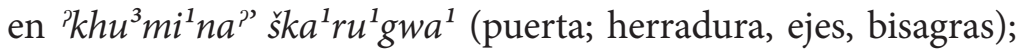
$\mathrm{Na}^{2}$ numina ${ }^{\text {?3 }} i^{1}$ š $^{\text {" }}$ "se está moliendo la madera (suena la matraca)".

Una fortaleza del alemán está en la formación de términos derivados en especial mediante proverbios: müffeln, "oler a algo desagradable"; erwittern, "descubrir con el olfato", auswittern, "oler de arriba a abajo"; abriechen, "obtener un olor tras mucho 
olfatear", beriechen, beschnuppern, beschnüffeln, "olisquear intensamente", herumschnuppern "olisquear por todos lados". También en wixárika se utilizan muchos términos derivados para sonidos, a pesar de que tienen un inventario muy amplio de términos básicos. Apestar y sobaquina son derivados. Los términos de color blancuzco, blanquecino, azulado y amarillento son derivados. Para otros colores o tonalidades se utilizan en huichol sobre todo derivados en -iye: haimaiye, "entre blanco y gris" (color de las nubes); tairaiye, "rosita"; taxaiye, "amarillo"; tsinakarimaitye, "verde limón".

Más descriptiva es la composición, que involucra dos lexemas. Es una técnica muy productiva en alemán, pero poco en castellano, wixárika y miphaa. Presentamos algunos ejemplos de esta última lengua. El verbo básico $g u^{3} w \tilde{\imath}^{33}$ significa "gemir por dolor físico o moral", mientras que $g u^{3} w \tilde{\imath}^{33} t h \tilde{\imath}$ se compone de $g u^{3} w \tilde{l}^{23}$ "gemir de dolor", y la palabra $e^{2} t h \tilde{l}^{1}$ "hablar, emitir voz"; el nuevo significado está convencionalizado como "mugir". $G a^{2} h a^{1}-m a^{21} h \tilde{a}^{1}$ "hervir intensamente, gluglutear", se compone de $g a^{2} h a^{1}$ "hervir" y $m a^{11} h \tilde{a}^{1}$ "dentro, en su interior". Škhuthu$m a^{2} h \tilde{i}^{1}$ ahngoo ${ }^{1}$ significa "cortar dentro voz, gorgoritear". Tha ra'ha "comunicarse a través de bocina, teléfono u otro aparato",

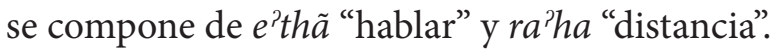

Un principio pragmático que subyace a todas las técnicas nominativas es que crean términos para entidades nombrables:

There are limits to what might count as nameable categories in general, and nameable derivational categories in particular. There are, however, no limits to the categories we can describe using syntactic structure. We can describe, syntactically, [...] someone as "a conservative, countryfied young woman of the upper or uppermiddle classes” (Bauer, 1988, p. 835).

Las técnicas léxicas establecen categorías de entidades nombrables, que se caracterizan por su permanencia en el tiempo y su relevancia cultural y discursiva. "Categories created by word-formation are presented as temporally unbounded [...] word-formation is not necessarily a suitable strategy for describing every new event o entity. Creating a new word creates a new 
category, describing something does not create a new category" (Bauer, 1988, p. 835).

La explicación de Bauer se formula como una oposición entre categorías "nombrables" y categorías "describibles" basada en la correspondencia con expresiones léxicas o morfológicas y expresiones sintácticas. Sería más adecuado hablar de un continuo que se extiende desde el estrato de los lexemas básicos hasta las expresiones más descriptivas. La composición está más cerca de la sintaxis que la derivación, pero menos que la incorporación, que a su vez presenta varias modalidades -léxica, sintáctica, discursiva- (ver Iturrioz, 2001).

Se podría hablar de una relación icónica, más exactamente diagramática, entre el grado de establecimiento sociocultural de categorías y las técnicas de nombramiento. Las categorías más conocidas, por mucho establecidas con firmeza, no necesitan ser descritas tan minuciosamente como las clases de referentes menos firme y por lo general establecidas. Las técnicas más descriptivas pueden ser elegidas para sonidos muy conocidos por razones de pragmática comunicativa; por ejemplo, cuando se trata de definir un término en el diccionario: las definiciones deben explicitar las propiedades implícitas en un término etiqueta. Por el contrario, no es factible inventar términos etiqueta cada vez que nos encontramos con un sonido desconocido.

Lo relevante es que se presupone una clase y que ésta debe ser estable, permanente en el tiempo, para poder ser una clase nombrable; es decir, digna de ser nombrada. Como ocurre con los nombres propios, que presuponen la existencia de individuos estables (no ponemos nombres propios a los rayos o a los pasos que damos al caminar), las clases también deben ser estables para que sean nombrables. No es una característica homogénea de todos los procesos de formación de términos, sino que depende de forma crucial del grado de nominalidad. En huichol, los nombres deverbativos en me que forman el plural añadiendo te no presuponen la existencia de una clase "nombrable" socialmente relevante; por ejemplo, xietemawaa-me-te, "enmieladores", o mematsi'utatiewaawe-me-te, "los que tienen la capacidad de insultarte", pero sí los que forman el plural eliminando el sufijo derivativo - $m e$ ante el flexivo de plural -te como mara'aakame - mara'aakáte, "chamanes", o tiyumíekame 
- tiyumieká-te, “cazadores”, los cuales preceden inmediatamente a los nombres propios en la escala de nominalización: Neikame (de nei, "brotar (el maíz)"); Xitákame (de xita, "jilotear (la milpa)”); en la cultura huichola, entre las clases socioculturales bien establecidas están la de los chamanes y los cazadores (de venados), pero no existe una clase de personas que se dediquen profesionalmente a enmielar y menos a insultar.

Todo esto es válido "en principio". Pero el hecho de que wixárika tenga tantos términos básicos para sonidos y miphaa tan pocos es una prueba de la insoslayable arbitrariedad de las lenguas. Las lenguas son un tipo de actividad orientada tanto por principios y estrategias generales cognitivos y de pragmática comunicativa, así como por sus derivas históricas.

\subsection{Técnica de etiquetado genérico y la regla de antonomasia}

Mi?phaa no tiene un término especial como ladrar, pero expresa esta idea mediante la combinación de un verbo genérico de emisión $w \tilde{a}^{1}$ "sonar, emitir su voz" con la designación de la fuente del sonido: na-wã $\tilde{a}^{1} \check{s} \mathcal{u} w \tilde{a}^{\text {?1 }}$ 'ATM-sonar perro' "suena el perro" significa "ladrar" a pesar de que los rasgos específicos de esta idea no están semantizados en ninguna de las dos palabras; ni $w \tilde{a}^{1}$ ni šu $\tilde{a}^{\text {?1 }}$ significan "ladrar"; el significado de la combinación no se agota en la semántica léxica. Para construir el significado completo, los hablantes/oyentes aplican una regla pragmática convencional que llamamos regla de antonomasia: Nawã $\tilde{a}^{1} \breve{u} w \tilde{a}^{\text {?1 }}$ designa la voz por antonomasia, característica o prototípica del perro. El significado no se puede transferir ni metaforizar porque no es inherente a las palabras. Si decimos nawa a ${ }^{1}$ aga ${ }^{1}$ no estamos afirmando que el cerdo ladre, sino que emite su voz característica que es gruñir. Esta técnica se aplica a casi todas las voces animales.

La expresión miphaa es semánticamente más económica sólo en el sentido de que la información que toma del léxico es menor ( $w \tilde{a}^{1}$ y $\left.\check{s} u w \tilde{a}^{\text {? }}\right)$, pero la regla pragmática detona en la combinación de ambas palabras componentes semánticos ausentes en las partes. La equivalencia global se debe a que la técnica genérica compensa el ahorro de información léxica con in- 
formación inducida del contexto por la mencionada regla. Si se tienen en cuenta los dos componentes complementarios de todo acto lingüístico, se entiende que nawa ${ }^{1} \check{s ̌ u w a}^{\text {?1 }}$ y el perro está ladrando son equivalentes. Pero el modus operandi de ambas lenguas es diferente. Nawã $\tilde{a}^{1} \check{\text { suwa }} \tilde{a}^{\text {?1 }}$ no presenta la misma redundancia que el perro está ladrando, porque los rasgos adicionales no se suman a la matriz de rasgos inherentes de $w \tilde{a}^{1}$, sino que son inferenciales y pertenecen a la expresión completa.

El significado de la combinación es más que la suma de los significados de sus componentes. Los rasgos específicos adicionales se deben a la regla pragmática que restringe la referencia a la voz por antonomasia de la especie perro. El significado de nawa $\tilde{a}^{1}$ šuwa $\tilde{a}^{21}$ no es composicional si entendemos que es la expresión equivalente a el perro / los perros están ladrando. En el dominio de los sonidos, castellano, alemán y huichol son lenguas inherenciales, mi phaa es inferencial. Mi’phaa economiza en el componente semántico, pero complejiza el componente pragmático, donde se crean inductivamente las lecturas específicas en dependencia de los contextos. La tendencia sintagmatizante y pragmatizante del mi'phaa se contrapone a la tendencia paradigmatizante y semantizante de las otras tres lenguas en el dominio de los sonidos.

Siguiendo el mismo esquema, nawã ${ }^{1} g u b o^{1}$ "suena rana" equivale a croar; nawã $\tilde{a}^{1} e^{1} d i$ "suena toro" equivale a mujir; nawã $\tilde{a}^{1} e k a$

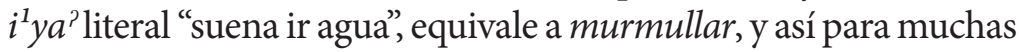
voces y sonidos. No se trata de polisemia. No es que $w \tilde{a}^{1}$ signifique "ladrar", porque entonces tendría que significar también "rebuznar", "relinchar", "croar", "barritar" y cualquier otro sonido cuya fuente se menciona. No hay solidaridad léxica como la hay entre ladrar y perro, rebuznar y burro, relinchar y caballo, $h w i^{1} \tilde{n} i^{1}$ y šuwa $\tilde{a}^{\text {?1 }}$. Se trata de una inferencia convencionalizada en reglas pragmáticas; la técnica de etiquetado genérico es la regla de antonomasia.

\subsection{Técnica resultativa y la regla de causalidad}

Si a un hablante de castellano se le pregunta cómo se llama el ruido que se hace al barrer, da respuestas como "no recuerdo", 
"no hay una palabra especial", "yo diría el ruido de barrer". En náhuatl, "barrer" se dice tla?ochpana y el ruido de barrer sasawaka. En huichol ocurre lo mismo: xuwanarika designa el ruido que hacen las escobas, mientras que 'itiya significa "limpiar"; derivados ambos de kixau "tostada", kixauni significa "estar seco (por inducción: crujiente)" y kixaurika significa "crujir". Un hablante de mi phaa dice: nawa $\tilde{a}^{1}$ gi ${ }^{1}$ ši "suena barrer" o simplemente nagi ${ }^{1}$ ši. Aunque todos los hablantes asocian ambas cosas cognitivamente, náhuatl y huichol tienen un verbo especial para el ruido que se produce al barrer (etiquetado específico), en castellano no hay tal verbo ni se emplearía barrer para suplirlo, y mi?phaa no tiene un verbo especial, pero recurre a otras técnicas para suplirlo como la seriación (nawa $\tilde{a}^{1} g i^{1}$ ši, "suena barrer") o la técnica resultativa $\left(n a g i^{1}{ }^{\wedge} i\right)$.

Notaat tla'ochpana? 'ikalmapa pampa 'onka miyak tlasolli $\quad$-inh $\quad$-inf $\quad$-conv Mi papá barre el patio de su casa porque hay mucha basura

Tlachwastli sasawaka? kema kiºchpana tlasolli +inh -inf -conv La escoba hace su ruido cuando barre la basura

Tlachwastli? kiwasaniya? tlalli? kema kiºchpana tlasolli $\quad$-inh $\quad$-inf $\quad$-conv La escoba raspa la tierra cuando barre la basura

Meta'itienitsie xuwanari ne'enieni. + inh -inf +conv Cuando comienzan a barrer se oye el ruido de las escobas

[inh $=$ inherente, inf $=$ inferencial, conv $=$ convencional $]$

El fundamento de esta técnica es una regla pragmática basada en una relación de causalidad entre eventos: un término que designa un proceso del cual resulta un sonido característico puede usarse para significar ese sonido. Esta técnica vincula dos ideas: la de un proceso causante (barrer) y la de un proceso resultante (ruido); el vínculo se establece por la vía de la pragmática (inferencia), no de la semántica (inherencia). Los diccionarios de castellano definen a veces chascar como "triturar, ronzar algún alimento quebradizo", y otras veces como verbo de sonido ("hacer ruido al triturar"), lo que pone de manifiesto la 
relación entre un proceso con el sonido que se produce en este proceso. Pero mientras aquí se trata de un proceso semántico, en tlapaneco es un asunto de pragmática.

En virtud de la estrecha asociación entre movimiento y sonido, el verbo špa $\tilde{a}^{1} g a$ "moverse" puede detonar una inferencia relativa al sonido:

(1) Khišpã ${ }^{1}$ ga ahwã?. "Se mueve metal (campana, herradura)".

Esta frase se emplea para expresar que la campana o la herradura floja se están moviendo. Como este movimiento va con frecuencia acompañado de sonido, por una metonimia convencional, špa $\tilde{a}^{1} g a$ se entiende como término de sonido. Las asociaciones inferenciales se originan en la coincidencia temporal reiterada que a su vez sirve de base a una relación de causalidad entre fenómenos. Cuando una tortilla se seca o se tuesta se hace quebradiza, produciendo un crujido al quebrarse. Hay una relación de causalidad entre quebrarse y crujir. De una relación entre las cosas y los procesos objetivos se pasa a inferencias convencionalizadas, y a significados léxicos cuando la lengua las convierte en rasgos inherentes, necesarios, implicados.

\subsection{Técnica del iconismo fónico}

Mientras en el etiquetado genérico se infiere el sonido a partir del conocimiento del animal, la técnica 9 consiste en combinar un verbo genérico con una expresión icónica de sonido que imita la voz o un ruido característico: mani $w a^{1} u$, "haré wau"; mani $k i^{1} k i^{1}$ rikiiii ${ }^{1}$ " "haré quiquiriquî". En mi'phaa se utiliza casi siempre el verbo genérico $e^{i} n i$, "emitir una voz o sonido". Compite con la técnica de etiquetado genérico para suplir la falta de términos básicos de sonido. A esta técnica se recurre con frecuencia en la literatura de ficción, donde las voces o sonidos imitativos resultan más efectivos que el vocabulario convencional.

(2) Mani wauuu, wauuu.

"Haré wauuu, wauuu". 
(3) Mani kikirikiii ${ }^{1} i^{1}$, kikirikiiii ${ }^{1} i^{1}-e^{2}$ thã mbašta ${ }^{1}$. "Haré quiquiriquí, dice el gallo".

(4) Mani iii ${ }^{1}$ iii $^{1}$ iii $^{1}$ iii $^{1}$ iiiiiiiiiiiii $^{1}$.

Voz del caballo.

Las voces icónicas forman un listado preléxico y pregramatical, pero de ellas derivan en wixárika numerosos lexemas adaptados a los patrones morfoléxicos del vocabulario convencional, donde el iconismo fónico decrece porque no se extiende a los morfemas derivativos y flexivos de la nueva palabra, porque las reglas de formación de sílabas se imponen. De $w u w u$ wu se forma wuwurika, que designa la voz del búho. De 'ui ${ }^{7} u i$

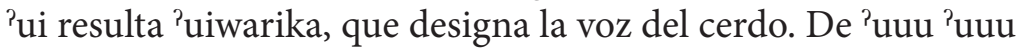
${ }^{2}$ uuu se forma ' $u^{\text {? } u w a r i k a, ~ q u e ~ r e m e d a ~ u n ~ g r i t o ~ d e ~ a n g u s t i a . ~ E n ~}$ wixárika estas voces van acompañadas a menudo de un verbo genérico de emisión o de percepción:

(5) 'Uuu, 'uuu 'uuu pireyu'eniwakai tewi.

“...se oía una persona” ('eni, "oír").

(6) Tuixu tsi riki ta waníu yapaukwa "'ui, 'ui" nepiretiniuni- tinehiawékaitini. "El cerdo inmediatamente contestó: 'diré ui ui”'.

(7) Kawaya waníu yapaukwa "híhihihihihiii" kanetayiní 'areutewimeki. "Caballo dizque en seguida relincho emitió prolongadamente".

Como en la técnica de etiquetado genérico, la inclusión de un término específico genera redundancia:

(8) Wau, wau nepiretihe'ewa kapá yiki peranuyeyaníki- tsiiki waníu netayiní. "Wau wau ladraré que no mal te pase perro dizque dijo".

En alemán y español las voces imitativas se toman como emisiones completas, que pueden ir seguidas directamente de las más diversas acotaciones:

(9) ¡Huyhuy! —suspiró encantado el silfo nocturno-.; ¿Un lugar maravilloso!

(10) Huuu! -seufzte der Nachtalb entzückt-. ;Eine wunderschöne Gegend!

\subsection{Técnica de seriación}

La técnica de seriación concatena en una expresión predicativa varios verbos sin nexos morfosintácticos aparentes: 
(11) Nawã ${ }^{1} e^{?} n i \quad h w i^{1} \tilde{n} i^{{ }^{1}} \stackrel{\text { šuwa }}{ } \tilde{r}^{11}$. Suena hacer gruñir perro.

(12) Nadža ${ }^{1}$ wũ $i w \tilde{a}^{1} e^{2}$ ni šti $i^{1} l a$.

Oigo sonar hacer gallina (oigo cacarear).

(13) Nadžawũ iwã ${ }^{1} e^{?}$ ni hwiñi ${ }^{11}$ na ${ }^{1}$ ša šuwã ${ }^{1}$.

Oigo sonar hacer gruñir rápido perro.

La seriación genera una redundancia creciente, ya que cada uno de los términos se asocia inferencialmente con el que sigue: džawũ, "oír", se infiere $w \tilde{a}^{1}$, "sonar", que a su vez se infiere de $e^{2} n i$, "emitir un sonido", que a su vez se infiere de $h w i^{1} \tilde{n} i^{1}$, y un término de cualidad de sonidos implica un predicado de sonido.

Los términos de la seriación se integran en una unidad funcional, donde na- se hace facultativo en todos los elementos, menos el primero: $n a-\left[w \tilde{a}^{1}+m i^{1} \tilde{n} \tilde{u}\right]$ šuwã ${ }^{1}$, "suena asustado perro"; na $\left[w \tilde{a}^{1} k i^{3} n a^{1}\right]$ šuwa $\tilde{a}^{\text {?1 }}$, "suena enojado perro".

(14) Nadžaawũ nawã $\tilde{a}^{1} n a^{p 1} n i$ gi ${ }^{1} n a^{1}$ šuwã $\tilde{r}^{\text {11 }}$.

Oigo suena hace triste perro.

(15) Nadžaawũ nawã ${ }^{1}$ nãdžahwa ${ }^{1} i^{1} n a^{1} \check{s ̌ u w a ̃ ~}^{\text {?1 }}$.

(16) Nadžaawũ nawã ${ }^{1} e^{2} n i$ mi $i^{1} \tilde{n} u^{1}$ šuwa $\tilde{a}^{11}$.

(17) Nadžawũ iwã $\tilde{a}^{1}$ na?ni hwiñi $i^{11}$ šuwã ${ }^{1}$.

Oigo sonar hace gruñir perro.

Los componentes de la serie no forman cláusulas independientes: no se puede intercalar $r{ }^{1}$ "lo que" sin que el verbo a su derecha deje de formar parte de la serie; el resultado es una cláusula relativa donde el verbo debe llevar el morfema $n a-$ : ${ }^{*} n a w \tilde{a}^{1} r^{1} e^{2} n i=>$ nawa ${ }^{1}{ }^{1} i^{1} n a^{2} n i$ "suena lo que hace", pero $n a^{2} n i$ se refiere entonces a cualquier acción acompañada o no de un sonido.

El primer elemento no puede llevar na- cuando va precedido de un término de cualidad:

(18) $\check{S} i^{1} g u\left(^{*} n a\right) d z ̌ a w u ̃ ~ i w a a^{1} e^{?} n i a^{2} d a^{1} \check{s ̌ u}^{1} w \tilde{a}^{?}$.

Agudo se oye sonar hacer niño perro (cachorro). "Agudos se oyen los gritos de un cachorro".

La seriación parece tener la función pragmática de anclar progresivamente el enunciado en la situación comunicativa identificando componentes del proceso como la audición, la emisión y 
la naturaleza dinámica del proceso mismo; este orden refleja una creciente relevancia semántica para el núcleo predicativo, mientras que en la dirección inversa aumenta la relevancia pragmática.

\subsection{Técnica analítica}

En tlapaneco la técnica analítica combina un término con especificaciones adicionales relacionadas con la acción que produce el sonido, con una cualidad inherente al sonido emitido, con una interpretación del sonido o con la intención comunicativa ligada a la emisión de la voz: nawãa $\tilde{a}^{1} i^{1} \tilde{n} \tilde{u}$ šuwa ${ }^{1}$ (amenaza); nawa ${ }^{1}$

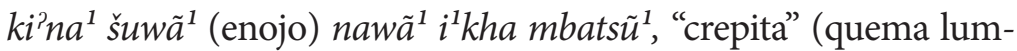
bre); nadžahwa ${ }^{1} i^{1} \eta \tilde{a}$ šuwã ${ }^{21}$, "grita triste perro" (aullar, otilar); naw $\tilde{a}^{1} e^{r 1} n i$ gahmi ${ }^{1} \check{s ̌}^{3} b u^{3}$, "se oye hacer protesta persona"; nawa ${ }^{1}$ $t s i^{1} m a^{1}$ štaa $m i^{1}{ }^{1}$ stũ , "se oye a gusto estar el gato, ronronea"; nawa ${ }^{1}$ $e^{i}$ tha $\tilde{a}^{1} \tilde{n} u^{\text {? }}$ u, "se oye hablar ave, crotorar (loro, cotorra, perico)"; nagu ${ }^{1} w \tilde{\imath}^{?}$ aga ${ }^{1} \check{s}^{1}{ }^{1} n a^{1}$ khumu ekhanu ${ }^{1} \check{s}^{3} a^{3} b u^{3}$, "gime jabalí siente llegar persona, rebudia"; naw $\tilde{a}^{1} e^{p 1} n i$ rũdup ${ }^{p 1} i^{1}$ do džah ũ $\tilde{a} d i \tilde{u}$, "se oye hace al pavo cuando llama a los suyos, tita"; šiši iwa $\tilde{a}^{1} e^{p 1} n i^{1}$ guma $n g u t s i$ / škwi $i^{1} y a$, "se oye hacer crujir tostadas / ramitas"; na $n i$ šraa ${ }^{3}$ $i^{1} x i$, "hace se resquebraja el árbol"; šraa $e^{3} n i i^{1}{ }_{s} i$, "crujiente hace el árbol"; šraa ${ }^{3}$ wa ${ }^{1} e^{2} n i i^{1}{ }^{1}$ si, "crujiente se oye hacer árbol”.

La técnica analítica es mínimamente convencional. La manera de expresar un concepto como chapotear no es constante, sino que adapta el grado de explicitud a situaciones comunicativas diversas.

Nawã ${ }^{1} k h i s ̌ p \tilde{a}^{1} g a h m a a n a k h u^{1} \check{s}^{3} a^{3} b u^{3}$. Se oye golpear con pies persona.

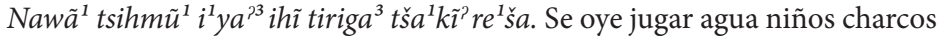
pequeños patio.

$A^{1} k h i$ iwa $\tilde{a}^{1}$ tsihm $\tilde{u}^{1} i^{1} y a^{{ }^{3}}$ ihĩ tiriga $a^{3}$ tš $^{1} k \tilde{l}^{2} r e^{1} \check{s} a$. Fuerte se oye jugar agua niños charcos pequeños patio.

\subsection{Perfil semántico pragmático de una palabra particular: ši $^{1} \mathrm{gu}$}

En este esquema representamos la estructura semántica y pragmática de una palabra particular. En el nivel 1 del esquema 
distinguimos tres acepciones, la primaria y dos analógicas relacionadas con patrones conductuales; la primera la traducimos con manorrota (se dice de una persona a la que todo se le resbala de la mano): $m i s i^{1} g u$ ñaw $\tilde{u}^{\text {?1 }}$, "de mano frágil"; la segunda la traducimos como quisquilloso, "demasiado delicado, que (se) agravia u ofende por cualquier causa"; miš $i^{1} g w i s ̌ a^{3} b u^{3}$, "una persona quisquillosa"; miši ${ }^{1} g u$ ahnga", "palabras mal intencionadas"; miši ${ }^{1} g u$ nithani, "lo hiciste con mala intención"; miši ${ }^{1} g u$ nirathã " "lo dijiste con mala intención".

(19) (Mi)ši $i^{1} g u e^{\text {?1 } n i ~ d z ̌ a ~}{ }^{1} k \tilde{u} \check{s} a^{3} b u^{3} t s u^{1} k h w i$.

"La plática de esa persona es quisquillosa".

Nos vamos a centrar en la acepción primaria. El significado primario de esta palabra se mantiene constante en los niveles inferiores, ya que se predica de manera general e irrefutable de todos los objetos que pertenecen a su dominio referencial, dividido en el nivel 2 en cinco subdominios que abarcan cinco clases o categorías de objetos de acuerdo con las diversas propiedades que propician los procesos respectivos que causan su fácil rompimiento. Se trata de cinco maneras diferentes de perder la unidad; la primera categoría se asocia con el proceso de triturar (machacar, ronzar, moler, chascar) con facilidad; para la segunda, la sequedad y la delgadez son los rasgos que causan su fácil rompimiento; para la tercera entra en consideración agrietarse (henderse, rajarse...), para la cuarta desmoronarse (pulverizarse, hacerse morusas), y para la quinta desprenderse (soltarse, arrancarse, desgranarse). En todos los casos hay que añadir "con facilidad". El dominio referencial de $\check{s}^{1} g u$ no es homogéneo, sino estratificado, con un prototipo que cumple plenamente con el significado (primera clase) y otras capas que se alejan gradualmente del mismo. No hablamos de cinco significados de $s i^{1} g u$, sino de cinco clases de objetos a los que se aplica de manera diferenciada. Si en el nivel 1 definimos el significado del término, en el 2, damos cuenta de la organización de su extensión o dominio referencial. 
1.1. $\breve{S} i^{1} g u$, "perder fácilmente la cohesión que constituye la unidad de un objeto". [2. ši ${ }^{1}$ gu ñawũ?, "manorrota". 3. $\check{s}^{1}{ }^{1} g w i$, "quisquilloso"].

Nivel 2.

\begin{tabular}{|c|c|c|c|c|}
\hline $\begin{array}{l}\text { Clase } 1^{1} \\
\text { triturar }^{6} \\
\text { gu }^{1} \mathrm{ma} \mathrm{ši}{ }^{1} \mathrm{gu} \\
\text { gu }^{1} \mathrm{ma} \text { ngutsi } \\
{\text { kafe } \mathrm{ni}^{1} \mathrm{gu}^{\text {? }}} \\
\text { eši }{ }^{1} \mathrm{ni}^{1} \mathrm{gu}^{\text {? }} \\
\text { pã ši }{ }^{1} \mathrm{gu}\end{array}$ & $\begin{array}{l}\text { Clase } 2^{2} \\
\text { aplastar > } \\
\text { ina }^{1} \text { nihndo } \\
\text { šoo }^{1} \text { šndu }^{1} \\
\text { štoo }^{1} \text { ya }^{1} h^{1} \\
\text { šta }^{1} \text { yaa } \\
\text { škwi } \\
\text { škwi }{ }^{1}{ }^{1} \text { y̌i }\end{array}$ & $\begin{array}{l}\text { Clase } 3^{3} \\
\text { agrietar > } \\
\text { ehwi } \\
\text { ndã }\end{array}$ & $\begin{array}{l}\text { Clase } 4^{4} \\
\text { desmoronar > } \\
\text { šndu hu }{ }^{3} \mathrm{ba}^{\text {?3 }} \\
\text { mbaa? } \\
\text { šndu su }{ }^{1} \mathrm{ca}\end{array}$ & $\begin{array}{l}{\text { Clase } 5^{5}}^{\text {desgranarse }} \\
\text { yaa }^{3} \\
\text { štoo }^{1} \mathrm{ya}^{1} \mathrm{ha}^{1}\end{array}$ \\
\hline
\end{tabular}

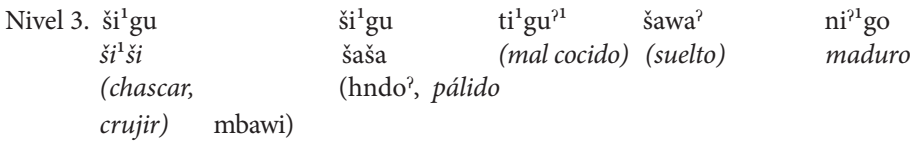

Nivel 4. $\quad$ agudo

Cuadro 5. Perfil semántico pragmático de ši ${ }^{1} \mathrm{gu}^{7}$

Si preguntamos a un hablante de castellano, alemán o wixárika, donde no hay un término con un significado equivalente, qué tienen en común las tostadas, hojas secas, vainas secas, comales de barro, ollas, terrones de azúcar, terrenos sueltos o mazorcas, las respuestas podrían ser muy variadas. Los hablantes de mi $i^{i}$ haa dan una respuesta inmediata y unánime: $\check{s}^{1} g u$ $n a h^{1} m a$, "todas pierden su integridad con facilidad". Su competencia semántica encuentra sin vacilación el significado que los une. Un hablante de castellano y un hablante de mi?phaa pueden

\footnotetext{
${ }^{1}$ En el orden de la lista: tostadas, doradas, café tostado, granos de maíz tostados, pan tostado, en este orden.

${ }^{2}$ Hojas secas, cáscara de huevo, corteza de frijol, corteza de árbol, varitas de árbol, varitas de arbusto, en este orden.

${ }^{3}$ Comal de barro, olla de barro, en este orden.

4 Terrón (bola de tierra), tierra, terrón de azúcar.

${ }^{5}$ Mazorca, corteza de frijol.

${ }^{6}$ Con los dientes, en el molino, en el metate.

${ }^{7} \check{s}^{1} g u$ es un término de cualidad cuando va precedido del modal mi-, y un término de proceso si va precedido del modal na-.
} 
tener el mismo conocimiento de los objetos, pero no hay coincidencia semántica. La rápida respuesta de los tlapanecos se debe sobre todo al conocimiento de la lengua, específicamente del significado constante de la palabra $\check{s}^{1} g u$.

No se trata de cinco significados alternativos de un $\check{s} i^{1} g u$ polisémico. $\breve{S} i^{1} g u$ significa en todos los casos "ser frágil, perder la unidad con facilidad". Se puede usar para decir que algunos objetos se trituran, aplastan, agrietan, desmoronan o desgranan, que están secos, delgados, mal cocidos, pálidos, sueltos o maduros, que hacen un ruido llamado ši ši o šaša, o que un sonido es agudo, pero nunca deja de significar "quebrarse con facilidad", pero la asociación con esos procesos es de orden inferencial. La competencia semántica sólo es una parte de la construcción del significado. La competencia pragmática complementa esta labor estableciendo una cadena de asociaciones inferenciales entre el significado básico y los procesos y las propiedades de las clases de objetos involucrados.

El hecho de que podamos traducir esta palabra con términos específicos para las diferentes maneras en que diferentes clases de objetos pierden la unidad (triturar, aplastar, agrietar, desmoronar, desgranar) no significa que ši ${ }^{1}$ gu tenga los significados de todos esos verbos, más bien los suple a todos. Como ocurre con $w \tilde{a}^{1}$, "sonar", tampoco aquí se trata de polisemia ni de diferentes ideas expresadas mediante un número considerable de términos homófonos: $\check{s}^{1} i^{1} \mathcal{u} 1, \breve{s} i^{1} g u 2 \ldots \check{s}^{1} g u$ n. Mientras en castellano se utilizan diferentes términos para los procesos por los que diversos tipos de objetos pierden la unidad o cohesión de las partes (no necesariamente con facilidad), es decir se lexematizan las diferencias entre estos procesos, en mi?phaa se usa un mismo término genérico para la idea que no se lexematiza en castellano, dejando al conocimiento del mundo y la capacidad inferencial del hablante la recuperación de información adicional a partir del contexto, de acuerdo con ciertas reglas pragmáticas convencionales.

Cada una de las categorías del segundo nivel se asocia con otros conceptos del tercer nivel que representan una condición necesaria para pertenecer a la categoría; se trata de propiedades de los objetos que desempeñan un papel importante en el siste- 
ma de inducciones que se activan en el discurso. $\check{S} i^{1} g u$ puede utilizarse para designar el sonido que resulta de los procesos o propiedades de las categorías 1 y 2, también puede utilizarse para decir seco y delgado, para expresar que un comal está mal cocido o que el barro está pálido, que un terrón es suelto o poco compacto y que una mazorca está madura y seca. De esa relación entre los términos del segundo nivel y los del tercero (chascar, mal cocido, suelto, seco o maduro) no se puede dar cuenta con las herramientas de la semántica (matrices de rasgos inherentes). En aplicación de una de las reglas pragmáticas que rigen la operación de aprehensión del sonido en mi'phaa, un verbo que designa un proceso del que resulta un sonido característico puede usarse para designar ese sonido. Así, el sonido que resulta de quebrarse con facilidad un objeto de la primera y segunda categorías se puede designar con $\check{s}^{1} g u$. Cuando una entidad de la categoría 1 se quiebra con facilidad $\left(\check{s}^{1}{ }^{1} g u\right)$ produce un sonido que se designa con el término básico más específico de sonido ši $i^{1}$ ši; en ši ${ }^{1}$ gu se asocian ambas categorías, $̌{ }^{1} i$ ši las separa. Por su parte, witha ${ }^{1}$ riga separa las categorías 1 y 2 , pero une las categorías 2 y 3 , separadas por $s i^{1} g u$ y $t i^{1} g u^{\text {? }}$, "mal cocido".

Si $\check{s}^{1} g u$ puede utilizarse para designar el sonido que resulta de los procesos de las clases 1 y 2, también puede utilizarse para decir seco y delgado, para expresar que un comal está mal cocido o que el barro está pálido, que un terrón es suelto o poco compacto y que una mazorca está madura y seca. De esa relación entre los términos del segundo nivel y los del tercero (chascar, mal cocido, suelto, seco o maduro) no se puede dar cuenta con las herramientas de la semántica (matrices de rasgos inherentes).

Finalmente, $\check{s i}^{1} g u$ llega a significar "agudo" (nivel 4). Este nuevo significado no deriva directamente de la definición básica, no se puede relacionar con ninguno de los rasgos inherentes: "romperse" o "con facilidad", sino que proviene de una de las dos primeras asociaciones pragmáticas que tienen que ver con sonido; el sonido que resulta de quebrarse los objetos de la categoría 1 no es agudo, se trata de una relación mediada por la cualidad "delgado" asociada con los objetos de la categoría 2, como las hojas secas. Es la situación típica de la polisemia. Este significado se hace independente de "perder la unidad con facilidad, ser 
frágil”, y ahora $\check{s}^{1}{ }^{1} g u$ se puede predicar de cualquier expresión de sonido sin tenerse que referir al mismo tiempo que algo se está quebrando, es un término general de cualidad de sonido, como lo es agudo en castellano, que no está restringido a sonidos producidos por objetos agudos.

En conclusión, mientras en castellano los conceptos específicos están codificados en el léxico, en detrimento de la unidad cognitiva que subyace a todos ellos, en mi'phaa se construyen como significados contextuales de una idea general, que es la única lexicalizada en $\mathrm{si}^{1} g u$. Donde castellano, alemán y sobre todo wixárika despliegan una considerable variación léxica, miphaa economiza en el dominio de la semántica léxica, pero complejiza el dominio de la pragmática, donde se crean inferencialmente las lecturas específicas en dependencia de los contextos. La tendencia sintagmatizante y pragmatizante del mi'phaa se contrapone a la tendencia paradigmatizante y semantizante de las otras tres lenguas.

\section{Referencias}

BAUER, L. (1988). Introducing Linguistic Morphology. Edimburgo: Edinburgh University Press.

Berlin, N. B. y Kay, P. (1969). Basic Color Terms. Berkeley: University of California Press.

Bruner, J. (1983). Child's Talk. Learning to Use Language. Nueva York: W.W. Norton.

Comrie, B. (1981). Language Universals and Linguistic Typology. Oxford: Blackwell.

Dressler, W. y Barbaresi, L. M. (1987). Elements of Morphopragmatics. Linguistic Agency. Universität Düsseldorf.

Dressler, W. y Barbaresi, L. M. (1993). Morphopragmatics. Berlín: Mouton de Gruyter.

Dressler, W. (1990). Morphopragmatics. Bulletin of the Language Institute of Gakushuin University, 13, 3-19.

Harweg, R. (1989). Is Coherence a Pragmatic Phenomenon? Comments on Dieter Viehweger's Paper 'Coherence -Interaction Modules’. En W. Heydrich, F. Neubauer, J. S. Petö- 
fi y E. Sözer (eds.), Connexity and Coherence (pp. 283-290). Berlín-Nueva York: W. de Gruyter.

ItURrioz LEZA, J. L. (1986). Teoría y método de UNITYP. En J. L. Iturrioz Leza y F. Leal Carretero (eds.), Memorias del I. Congreso Interamericano de Filosofía (pp. 7-78). Guadalajara: Universidad de Guadalajara, México.

ITURRIOz LEZA, J. L. (1998). El acoplamiento estructural y la adquisición del huichol como lengua materna. Función, 17, 1-140.

ItURrioz LeZA, J. L. (2001). Inkorporation. En M. Haspelmath et al., Language Typology and Language Universals. Serie Handbücher zur Sprach- und Kommunikationswissenschaft 20.1 (pp. 714-725). Berlín: Walter de Gruyter.

ItUrRioz LezA, J. L. (2013). Desarrollo de la cohesión textual en la canción huichola. En B. Pérez Alvarez (coord.), Procesos de cohesión textual. Estudios de huichol y español (pp. 71106). Morelia: Universidad Michoacana de San Nicolás de Hidalgo.

LUNDQUIST, L. (1991). La cohérence textuelle révisée: une étude pragmatique. Folia Linguistica, 25(1-2), 91-110.

Mithun, M. (1984). The Evolution of Noun Incorporation. Language, 60, 847-893.

Piaget, J. (1974). Adaptation vitale et psychologie de l'intelligence. París: Hermann.

Piaget, J. et al. (1984). Los procesos de adaptación. Buenos Aires: Nueva Visión.

SeIler, H. (1976). Determination: A Universal Dimension for Inter-Language Comparison. Arbeiten des Kölner Universalien-Projekts, Nr. 23.

Tomasello, M. (2002). Constructing a Language: A UsageBased Theory of Language Acquisition. Harvard: Harvard University Press.

Viehweger, D. (1989). Coherence is Also a Pragmatic Phenomenon. Some Rejoinders to E.-M. Conte and R. Harweg. En W. Heydrich, F. Neubauer, J. S. Petöfi y E. Sözer (eds.), Connexity and Coherence (pp. 291-302). Berlín-Nueva York: W. de Gruyter. 